\title{
Deployment of Proposed Botnet Monitoring Platform using Online Malware Analysis for Distributed Environment
}

\author{
Vidhya Sathish* and P. Sheik Abdul Khader \\ Department of Computer Applications, B. S. Abdur Rahman University, Chennai, Tamil Nadu, India; \\ vidhyasathish83@gmail.com, psakhader@bsauniv.ac.in
}

\begin{abstract}
The main intention of this paper is to elaborately discuss about seriousness of Botnet problem and project the importance of online malware analysis in Botnet defense research.
\end{abstract}

Keywords: Botnet, Honeypot, IDS, Malware Analysis, OpenDNS

\section{Introduction}

Botnet $^{1,2}$ acts as a base for many illicit works, according to cyber community. Botnet has been categorized into centralized, decentralized and hybrid structures. In Centralized Structure ${ }^{3}$, one or group of compromised client machine will be remotely controlled by single server: Examples of Centralized Structure are IRC-based and HTTP-based. IRC-based Botnet is the oldest method followed by hacker. Main feature is it acts as communication protocols to reroute with compromised networks. Many defensive techniques have been proposed. Behalf of this, these communication protocols has been isolated from normal traffic. But IRC [Internet-Relay-Chat] based Botnet is still exists. Later, Hacker developed HTTPbased Botnet to achieve the destination by disrupting defensive techniques which was built against them. The main feature of HTTP-based Botnet is to hide from the users using the concept of dynamic domain name service, as a resolution to update and frequently changes the server location. In Decentralized Structure ${ }^{4}$, each Bot [compromised machine] acts as client and server. There is no centralized point of failure in such approaches. Examples of Decentralized Structure are P2P-based and fast-flux-based. These two approaches use the concept of fully qualified dynamic domain name service [FQDDNS] for frequent updates of new Botnet in automate manner. Many detection approaches are still being developed recent years. But it finds very difficult in shutdown and disrupt the Botnet resilience. Hybrid structure involves the combination of both centralized and decentralized structure. Recently, these types of structures are used as deliberate to spread Botnet in distributed environment. Based on History of Botnet, Figure 1 provides the complete structure of problem overview and detection approaches used.

\subsection{Scope of Research Work in Botnet Defense}

A lot of researchers engaged with the new techniques ${ }^{5}$ in recent years. But, advancement of Botnet defense is limited in scope. Some of them quietly impressed with Honeypot technology in Botnet defense research. Intrusion Detection/Prevention systems also plays effective role in understanding Bot and Botnet behavior. Finally, malware analysis also takes part as a major key role to identify the intention of attacks and also about hacker's nature. The concept of this paper is to provide proposed framework

${ }^{*}$ Author for correspondence 


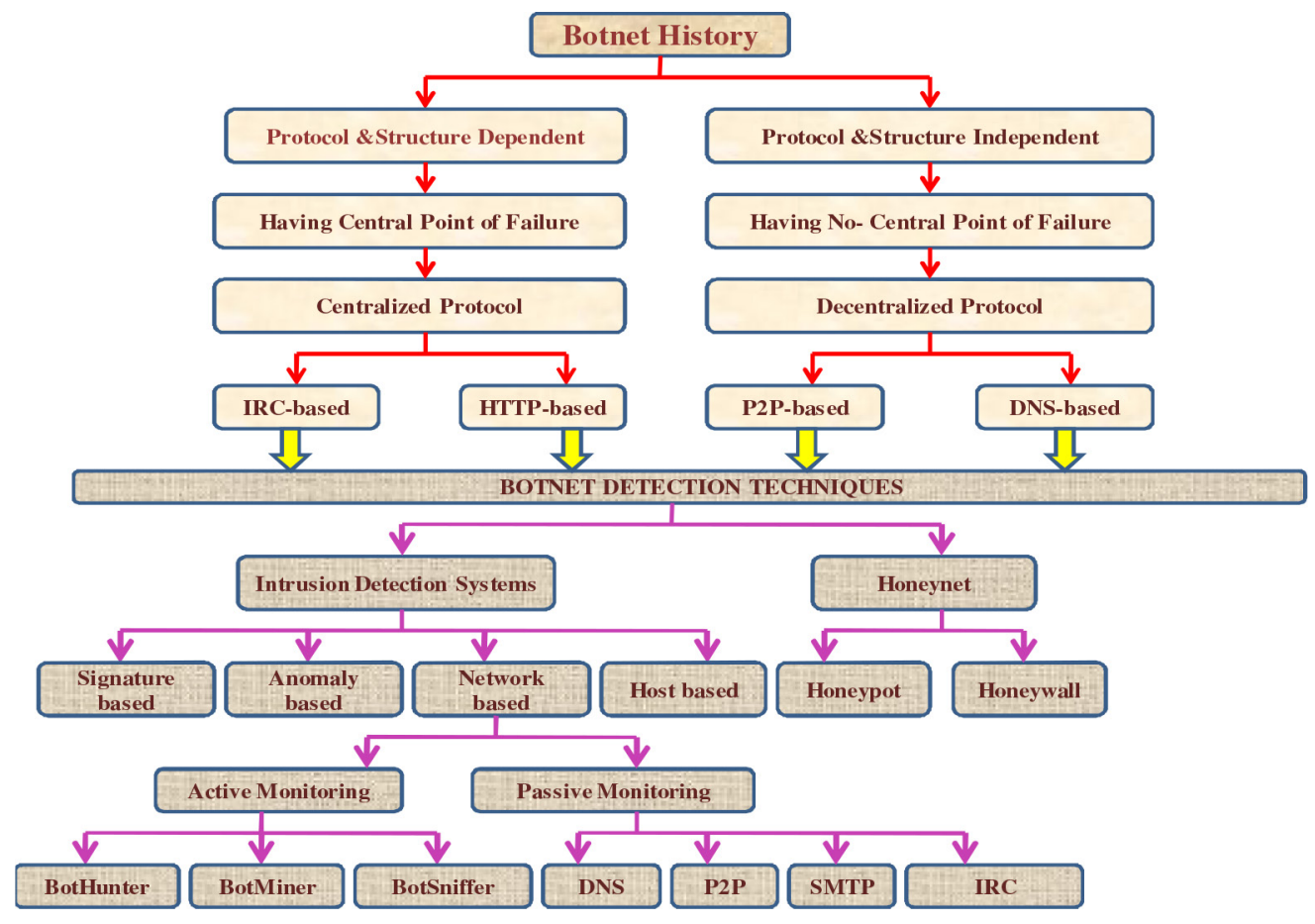

Figure 1. Botnet History and its Detection approaches.

by integrating Honeypot, Intrusion Detection Systems and malware analysis with aim to study Bot malware behavior and its nature in better phenomenon.

In this paper section II provides brief history about Honeypot technology, Intrusion Detection Systems and malware analysis importance in Botnet defense. Section III provides the related works done in Botnet mitigation and defense research. Section IV discusses about research directions in Botnet Defense. Section V presents detailed description of proposed framework using online malware analysis. Section VI concludes the future of proposed framework.

\section{Brief History}

\subsection{Honeypot Technology}

It is a technology $y^{6,7}$ designed to trap attackers to study their intention and behavior in automated manner. In other words, Honeypot is designed as a decoy system with an isolated environment to make the attackers voluntarily interact with them without their intention of that their idea is being trapped by an isolated source. The main ethic of this technology is data capturing; monitoring and analyzing the malware behavior. Another merit is, based on the user convince Honeypot can be deployed in both physical and virtual environment.

\subsection{IDS}

Intrusion Detection Systems ${ }^{8}$ able to handle various kinds of techniques and methods to identify abnormal malicious activities found in the network. The main ethic of IDS is to monitor the network traffic and analyze it, if any suspicious file present. It may also notify the presence of signature-based and anomaly-based attacks. Another merit is easy to deploy and maintain and also provide the real-time alert to novice users.

\subsection{Malware Analysis}

Malware analysis ${ }^{9-11}$ is most attractive topic for lot of researchers recent days. The 'malware analysis' is to study malicious piece of program and also makes the users to understand the malware behavior with deep inspection in an isolated environment i.e., why, when, where it should be reached. Generally, observing real-time network traffic is most challenging task. But, through malware analysis it is possible to achieve this task without affecting the system.

\section{Related Works}

Botnet detection and mitigation has been classified as Intrusion Detection Systems based, Data Mining based and DNS based. 


\subsection{IDS based Detection}

IDS $^{12-15}$ are generally classified into signature-based and anomaly-based intrusion detection systems. In Signaturebased approach, the detection systems able to capture and log malicious traffic based on rules and signatures generated already i.e., able to capture only known malicious activity. In Anomaly-based approach, the detection systems depend on network traffic anomalies present in it. Anomaly based further classified into Host-based and Network-based. In Host-based, network traffic packets analyze both known and unknown suspicious files. In Network-based, capture and analyze the data to detect known attacks by comparing the signatures or patterns of database or detection of illegal activities by scanning traffic for anomalous activity. The major drawback of these approaches is they seem to be protocol structure and dependent and also these are fails to analyze malware behavior and nature.

\subsection{Data Mining based Detection}

The main purpose of this type of detection ${ }^{16,17}$ is used for optimization. It has been further classified into flowcorrelation algorithm, classification algorithm, and clustering and finally association rule. In Flow-correlation algorithm, useful to compare flow objects based on some characteristic other than packet content. This technique is very effective and utilizes the characteristic values as input into one or more functions to create metric used to decide if flows are correlated. In classification algorithm, incoming packet will match one of previous patterns. The major limitation is, it is not appropriate approach to detect new attacks. In clustering, divide entire set data into subgroups or clusters containing relatively identical features and limitation is it does not require a labeled dataset for training. In association rule based, derive the implication relationship between data items under the conditions of set of given project types and number of records and finally analyzing the records. The major drawback behind is no real-time detection; protocol structure and dependent and also no proof of utilizing malware analysis to overcome Botnet resilience.

\subsection{DNS based Detection}

This technique ${ }^{18,19}$ identifies key metrics for measuring Botnet utility and describes various topological structures that BOTNET may use to coordinate attacks.. The limitation of this approach it could be evaded if Botmasters know the mechanism or suspect it is running. Botmaster may also poison the scheme through 'fake DNS' thus generating many false alarms. Another approach is DNS based BlackHole List (DNSBL) used to publish the addresses of computers or networks linked to spamming and other malicious activities. The major limitation is approach is not effective because it is not difficult to design evasion strategies.

\section{Research Directions in Botnet Defense}

Most of Detection approaches fail ${ }^{20}$ to analyze the malware to study its nature and behavior. Moreover analyzing data in real-time is tend to be most tedious task; also disruption of Botnets is lack of efficient techniques. Most of malware ${ }^{21}$ used by Botnets "runs only" on MS Windows, making - "ms windows machines the main targets". More advanced Botnet technique at present is DDNS [Dynamic Domain Name Service]- also known as fast-flux [Bots would query certain domain i.e., mapped onto IPADDRESSES, which change frequently]. Fast-flux makes Botnet more difficult to takedown or blocks a specific $\mathrm{C} \& \mathrm{C}$ server. Research on Botnet is relatively new and it has been subject of increasing interest in recent years. Existing studies remain limited in scope do not include recent research \& developments. It is necessary to analyze a massive amount of data, which is difficult to perform in real-time thus making detection in large-scale networks a prohibitive task. The sandboxed training environment ${ }^{22}$ can be improved to better circumvent malware authors to probe for virtual machine settings and react by stopping all activity. Creating "real-time Botnet Monitoring platform" and identifying new Bot variants and developing network sandboxing mechanisms that prevent captive Bot nodes from causing them.

\section{Proposed Framework for Online Malware Analysis}

Proposed framework has been designed based on descriptive architecture of Botnet problem. It paved the new way of research to study and analyze the malware behavior. As reviewed from lot of journals related to Botnet defense research, utilizing the DNS Sinkhole server for malware analysis is not in a sufficient way for research work. Based on this, proposed work has taken as a challenge to use this task for online malware analysis. 
In proposed work, virtual testbed will be created using vmware workstation in windows 7 Host machine. Figure 2 of results shows desktop display of Windows 7 in virtual machine. The major reason to choose windows operating system as platform to work with proposed, because MS windows machines are main targets for most cyber attacks. Benefit of vmware is able to create one or more virtual machines within single system. It will be treated as guest machine. Additional tools used for proposed work are listed below:

\subsection{Opendns}

Opendns servers ${ }^{23}$ store the ip addresses of millions of websites in their cache. It works in cloud to track malicious traffic and blocks phishing websites from loading the system. In Figure 3 of results show the utilization of opendns for internet access. Also, Figure 4 of results

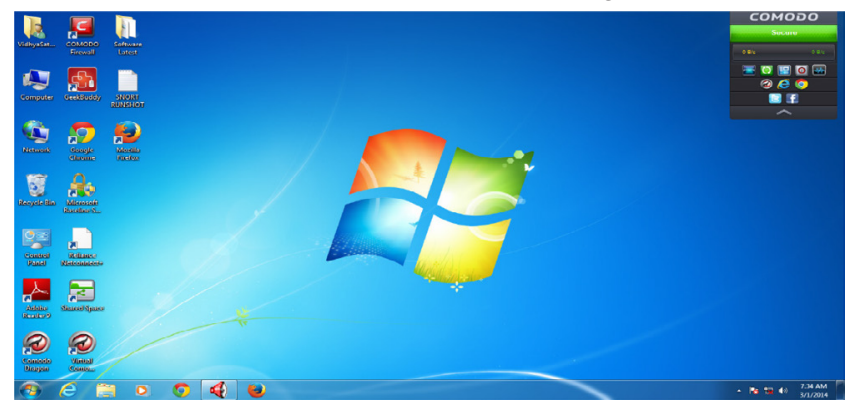

Figure 2. Desktop display of virtual machine.

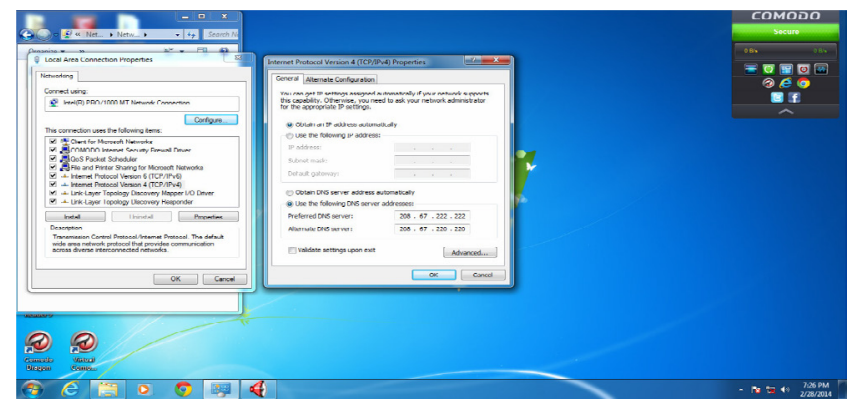

Figure 3. Utilizing opendns for internet access.
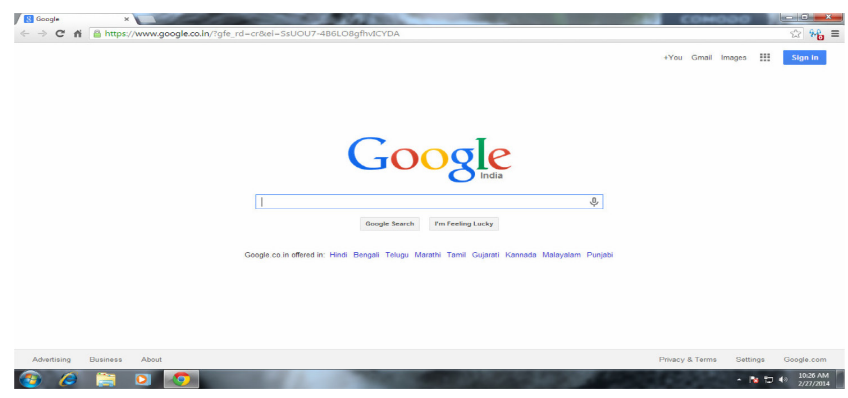

Figure 4. Homepage using opendns. shows the homepage access. OpenDNS runs a high-performance network which is geographically distributed and serviced by several redundant connections. Table 1 shows the comparative study of DNS services.

\subsection{KFSensor}

Windows based Honeypot Intrusion Detection Systems is a simulation tool ${ }^{24}$ act as Honeypot to attract and detect Hackers and worms by simulating vulnerable system services and Trojans. By acting as decoy server it can divert attacks from critical systems and provide a higher level of information than can be achieved by using firewall and NIDS alone. From the Figures 5 and 6 of results shows the normal and abnormal behavior of network activity in opendns network traffic. The system is highly configurable and features detailed logging, analysis of attack and security alerts. This approach complements other forms of security and adds another defense against growing security threat faced by organization.

Table 1. Comparing DNS services

\begin{tabular}{lccc}
\hline & $\begin{array}{c}\text { Comcast } \\
\text { AT\&T }\end{array}$ & $\begin{array}{c}\text { Google } \\
\text { publicDNS }\end{array}$ & $\begin{array}{c}\text { OpenDNS } \\
\text { Basic }\end{array}$ \\
\hline $\begin{array}{l}\text { Servers located } \\
\text { world-wide }\end{array}$ & & yes & yes \\
DNS security & maybe & yes & yes \\
IPV6 support & & & yes \\
Domain & & yes \\
category & & & yes \\
Tagging & & yes \\
Filter & & \\
unwanted sites & & &
\end{tabular}

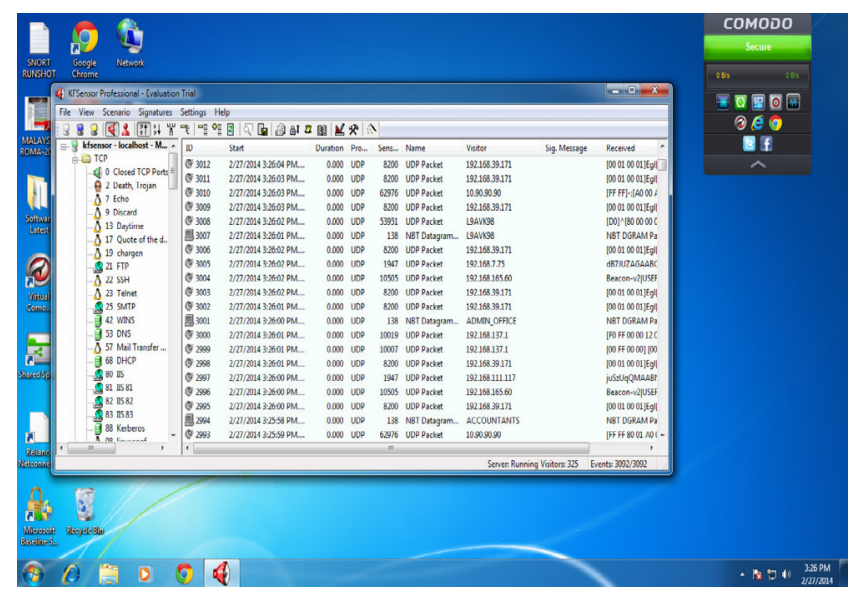

Figure 5. Normal behavior of opendns network traffic. 


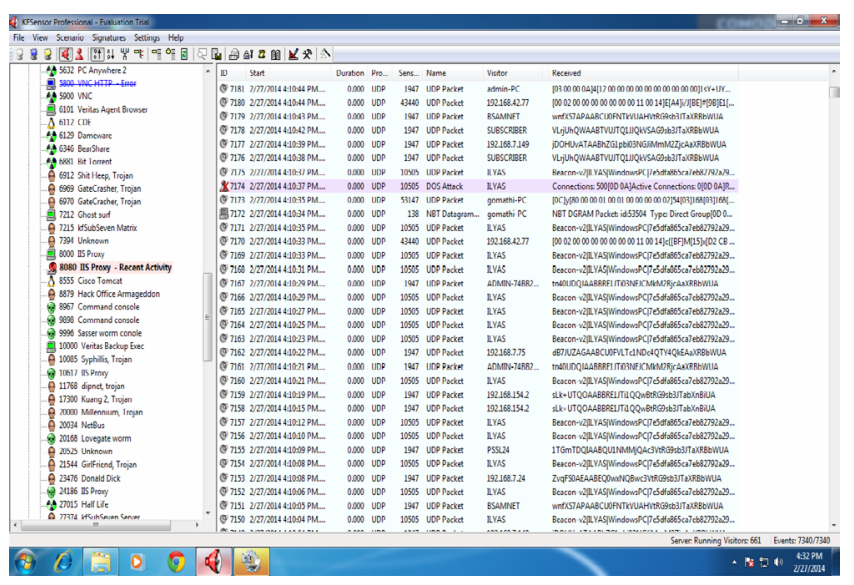

Figure 6. Abnormal behavior of opendns network traffic.

\subsection{SnortIDS}

SnortIDS ${ }^{25}$ is an open source network intrusion detection/prevention system. Used as real-time in proposed system. Snort can be configured in three main modes: sniffer, packet logger, and network intrusion detection. In sniffer mode, the program will read network packets and display them on the console. In packet logger mode, the program will log packets to the disk. In intrusion detection mode, the program will monitor network traffic and analyze it against a rule set defined by the user. From the Figure 7 of results shows the snort running for real-time alert in virtual machine. The program will then perform a specific action based on what has been identified.

\subsection{COMODOfirewall}

Comodo firewall ${ }^{26}$ is really good personal firewall which has several configurable features that makes use of latest technologies like 'Host based IDS' for actively monitoring and protecting your system from multiple type of attacks. The merit of comodo is plays a major role in maintaining the functionality and usability of system. It also prevents network-based attacks from the system.

\subsection{Netflow Analyzer-Manage Engine}

Used as network bandwidth ${ }^{27,28}$ monitoring tool in proposed work to provide holistic view about network bandwidth and traffic patterns. ManageEngine NetFlow Analyzer is a web-based bandwidth monitoring tool that collects, correlates, and analyzes NetFlow versions exports to show you what applications are using bandwidth, who is using them, and for how long. View in-depth bandwidth reports across your WAN and LAN without having to deploy expensive hardware probes. Recognize most enterprise applications and see how traffic flows across your network. NetFlow Analyzer also monitors critical VoIP metrics.

\subsection{Fakenet}

FakeNet ${ }^{29}$ is a tool that aids in the dynamic analysis of malicious software. The tool simulates a network so that malware interacting with a remote host continues to run allowing the analyst to observe the malware's network activity from within a safe environment. The main features are being easy to install and use; the tool runs on Windows and requires no 3rd party libraries. Support the most common protocols used by malware. Figure 8 of results shows the fakenet observation in opendns from the virtual machine. Perform all activity on the local machine to avoid the need for a second virtual machine. Provide python extensions for adding new or custom protocols. Keep the malware running so that you can observe as much of its functionality as possible. Have a flexible configuration, but no required configuration

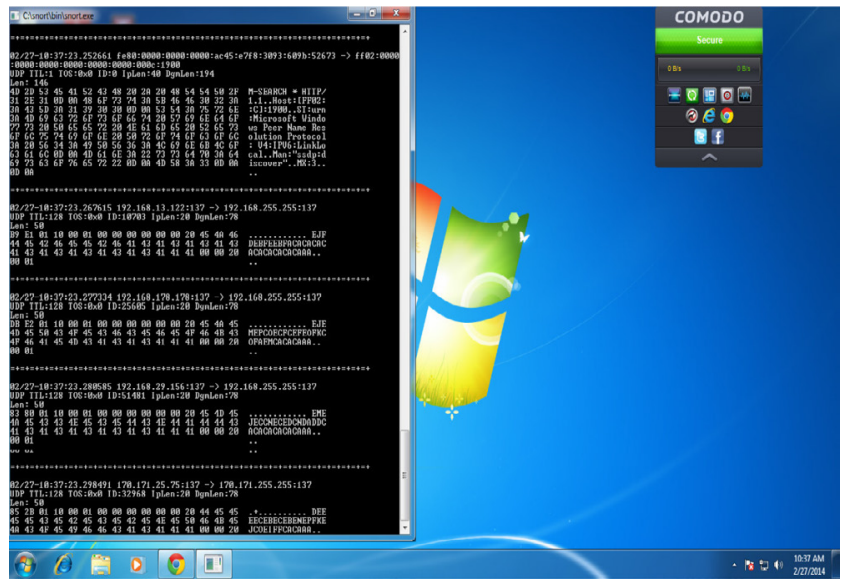

Figure 7. Running snort for real-time alert.

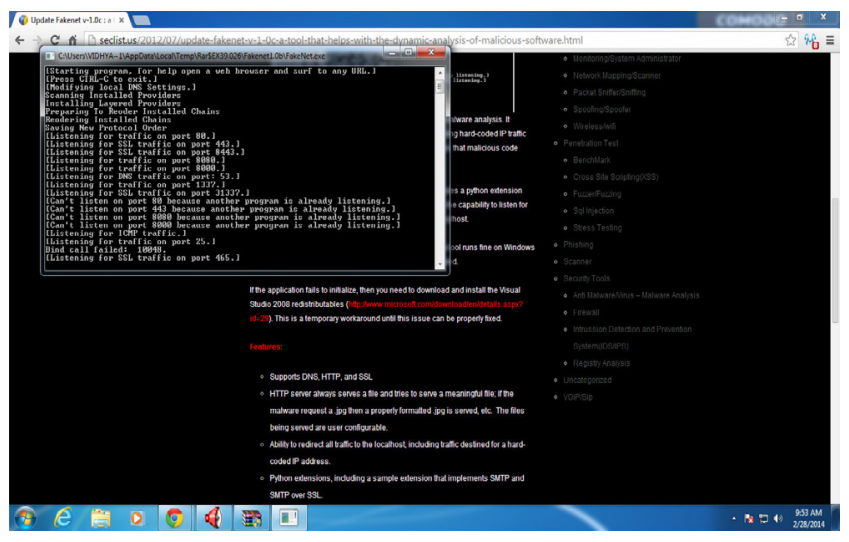

Figure 8. Observation of fakenet in opendns. 


\subsection{Capture BAT}

This is a behavioral analysis tool of applications for the Win32 operating system family. Capture BAT ${ }^{30,31}$ is able to monitor the state of a system during the execution of applications and processing of documents, which provides an analyst with insights on how the software operates even if no source code is available. Known event noise can be excluded by a fine-grained mechanism that allows an analyst to take into account the process that cause the various state changes. As a result, this mechanism even allows Capture to analyze the behavior of documents that execute within the context of an application, for example the behavior of a malicious Microsoft Word document.

\section{Results}

The experimental setup has been created using VMware workstation in Windows 7 operating system. The proposed work utilizes the openDNS sinkhole system to have real-time observation in network traffic. Also utilize the supporting tools to make the observation accuracy such as KFsensor, SnortIDS, and Fakenet etc. some of the screenshots of proposed work listed below.

\section{Conclusion}

We presented an approach for effective Botnet defense by utilizing the DNS sinkhole for online analysis. The proposed has been designed by integrating Honeypot technology, Intrusion Detection Systems and malware analysis in Windows based platform for Botnet research. We also discussed about research limitations in Botnet defense. In future, it can be employed offline using python based forensic analysis.

\section{References}

1. Zhu Z, Lu G, Chen Y. Botnet research survey. Annual IEEE International Computer Software and Application Conference, IEEE Computer Society. 2008.

2. Gross B-S, Cova M et.al. Your botnet is mybotnet: analysis of a botnet takeover. CCS'09, Nov 9-13 2009, ACM.

3. Li C, Jiang W, Zou X. Botnet: survey and casestudy. In 4TH International Conference on Innovative Computing, Information \& Control, IEEE Computer Society; 2009; IEEE.

4. Feily M, Shahrestani A et al. A survey of Botnet \& Botnet detection. 2009 3rd International Conference on Emerging
Security Information, Systems \& Technologies IEEE Computer Society; 2009; IEEE.

5. Silva SSC, Silva RMP et al. Botnets: A Survey. Computer Networks. 2013; 57:378-403.

6. Tiwari R, Jain A. Improving network security and design using honeypots. CUBE; 2012 Sep 3-5.

7. Pham V-H, Dacier M. Honeypot trace forensics: the observation viewpoint matters. Future Generation Computer Systems Elsevier; 2010.

8. Raghava NS, Sahgal D, Chandna S. Classification of botnet detection based on botnet architecture. 2012 International Conference on Communication Systems \& Network Topologies, IEEE Computer Society. 2012

9. Kasama T, Yoshika $\mathrm{K}$ et al. Malware sandbox analysis with efficient observation of herder's behavior. J Inform Secur. 2012 Oct; 20(4):835-45.

10. Egele M, Scholte T, Kirda E, Kruegel C. A survey on automated dynamic malware analysis techniques \& tools. ACM Computing Surveys. 2012; 1-49.

11. Graziano M, Leita C, Balzarotti D. Towards network containment in malware analysis systems. ACSAC'12, 2012 Dec 3-7.

12. Goebel J, Holz T, Rishi. Identify Bot contaminated hosts by IRC nickname evaluation. Proceedings of the first conference on First Workshop on Hot Topics in Understanding Botnets, USENIX Association, Berkeley, CA, USA; 2007.

13. Liu L, Chen S, Yan G, Zhang Z. BotTracer: Execution-based Bot like malware detection. Wu T, Lei C, Rijmen V, Lee D, editor. Information Security, Lect Notes Comput Sci. 2008; 5222: 97-113.

14. Xu K, Yao D, Ma Q, Crowell A. Detecting infection onset with behavior-based policies. 5th International Conference on Network \& System Security (NSS); 2011. p. 57-64.

15. Gu G, Yegneswaran V, Porras P, Stoll J, Lee W. Active Botnet Probing to Identify Obscure C\&C Channels. Computer Security Applications Conference, ACSAC'09; 2009. p. 241-53.

16. Gu G, Porras P, Yegneswaran V, Fong M, Lee W. BotHunter: Detecting Malware Infection Through IDS-Driven Dialog Correlation. in: proceedings of 16th USENIX Security Symposium on USENIX Security Symposium, USENIX Association, Berkeley; 2007; CA: USA. p. 12:1-12:6.

17. Gu G, Perdisci R, Zhang J, Lee W. BotMiner: Clustering Analysis of Network Traffic for Protocol \& Structure Indepdenent Botnet Detection. Proceedings of 17 th Conference on Security Symposium, USENIX Association; 2008; Berkeley, CA: USA. p. 139-54.

18. Choi H, Lee H, Kim H. BOTGAD: Detecting Botnets By Capturing Group Activities in Network Traffic. Proceedings of the fourth International Conference on Communication System Software \& Middleware, COMSWARE'09; 2009; New York: USA. p. 21-8. 
19. Villamerin-Salomon R, Brustoloni J. Identifying Botnets using Anomaly Detection Techniques Applied to DNS Traffic. 5th IEEE Consumer Communications and Networking Conference, CCNC 2008; 2008. p. 476-81.

20. Zhuge J, Holz T, Han X et al. Collecting Autonomous Spreading Malware using High-Interaction Honeypots. ICICS 2007, LNCS 4861; 2007; Springer-Verlag; Berlin Heidelberg 2007. p. 438-51,

21. Tegeler F, Fu X, Vigna G, Kruegel C. Botfinder: Finding Bots In Network Traffic Without Deep Packet Inspection. Co-NEXT'12; 2012 Dec 10-13.

22. BrettStone-Gross, Marco Cova, Bob Gilbert et.al. Analysis of a botnet takeover. IEEE Computer and Reliability Societies; 2011. p. 1540-7993, IEEE 2011.

23. Available from: www.404techsupport,com/2010/02/ opendns-pt-2-a-comparison/

24. Available from: www.highbeam.com/doc/1G1.101853773. html/
25. Chakraborti S, Chakraborty M, Mukhopadhyay I. Study of Snort-based IDS. Proceedings of International Conference \& Workshop on Emerging Trends in Technology; 2010. p. $43-47$.

26. Available from: www.ida.liu.se/projects/firewallcomparison/infosecpaper.pdf

27. Available from: www.manageengine.com/products/netflow/manageengine-netflowanalyzer.pdf

28. Available from: www.manageengine.com/products/netflow/netflow-features.html

29. Available from: www.practicalmalwareanalysis.com

30. Available from: www.honeynet.org

31. Watson D, Riden J. The honeynet project: data collection tools, infrastructure, archives and analysis. WISTDCS '08. WOMBAT Workshop on Information Security Threats Data Collection and Sharing, 2008; 2008 Apr 21-22. p. 24-30. 